Editorial

\title{
Acknowledgment of Reviewers of Reactions in 2021
}

\author{
Reactions Editorial Office
}

Citation: Reactions Editorial Office. Acknowledgment of Reviewers of Reactions in 2021. Reactions 2022, 3, 138. https://doi.org/10.3390/ reactions 3010010

Published: 29 January 2022

Publisher's Note: MDPI stays neutral with regard to jurisdictional claims in published maps and institutional affiliations.

Copyright: (C) 2022 by the author. Licensee MDPI, Basel, Switzerland. This article is an open access article distributed under the terms and conditions of the Creative Commons Attribution (CC BY) license (https://creativecommons.org/licenses/by/4.0/).
MDPI AG, St. Alban-Anlage 66, 4052 Basel, Switzerland

Rigorous peer reviews are the basis of high-quality academic publishing. Thanks to the great efforts of our reviewers, Reactions was able to maintain its standards for the high quality of its published papers. Thanks to the contribution of our reviewers, in 2021, the median time to first decision was 19 days, and the median time to publication was 47 days. The editors would like to extend their gratitude and recognition to the following reviewers for their precious time and dedication, regardless of whether the papers they reviewed were finally published:

\begin{tabular}{|c|c|}
\hline A. J. Romero-Anaya & Martyna Dymek \\
\hline Aitor Arregi & Miguel Menéndez \\
\hline Alberto Marra & Miriam Gonzalez Castano \\
\hline Alessandro Di Michele & Mohammad Taherzadeh \\
\hline Alexander Hansen Bork & Mohammadreza Shokouhimehr \\
\hline Alexey V. Ivanov & Morris Argyle \\
\hline Ana María Díez-Pascual & Muhammad Arshad \\
\hline Anand Chokkalingam & Nebojsa Marinkovic \\
\hline Andrey Khalimon & Niclas Solin \\
\hline Antonio Valverde-González & Omid Akhavan \\
\hline Dong-Pyo Kim & Paolo Defilippis \\
\hline Emilio Muñoz-Sandoval & Pierre Vogel \\
\hline Enrique Aguilar & Ranjith Thangavel \\
\hline Esmail Doustkhah & Robert C. Bowman \\
\hline Gianvito Vilé & Sandie Dann \\
\hline Hendrik Kosslick & Sebastiano Campisi \\
\hline Hugo de Lasa & Sergei Chernyak \\
\hline Ismael Matino & Seung Wook Kim \\
\hline Jae-hyeok Shim & Sónia Carabineiro \\
\hline Jiahan Xie & Sukeun Yoon \\
\hline Joana Alves & Thuat Trinh \\
\hline József S. Pap & Tiago A. Fernandes \\
\hline Kamila Sadowska & Tomasz Czujko \\
\hline Ken Sakata & Vassilios Dracopoulos \\
\hline Kollarigowda Ravichandran & Vega Lloveras \\
\hline M. R. M. Asyraf & Wanda Ziemkowska \\
\hline Maria Elena Russo & Xiaochen Shen \\
\hline
\end{tabular}

Maria Magomedova

\author{
Martyna Dymek \\ Miguel Menéndez \\ Miriam Gonzalez Castano \\ Mohammad Taherzadeh \\ Mohammadreza Shokouhimehr \\ Morris Argyle \\ Muhammad Arshad \\ Nebojsa Marinkovic \\ Omid Akhavan \\ Paolo Defilippis \\ Pierre \\ Sandie Dann \\ Sebastiano Campisi \\ Sergei Chernyak \\ Seung Wook Kim \\ Sónia Carabineiro \\ Sukeun Yoon \\ Thuat Trinh \\ Tiago A. Fernandes \\ Tomasz Czujko \\ Vassilios Dracopoulos \\ Vega Lloveras \\ Xiaochen Shen
}

